\title{
Isolation and Identification of Lipase Activators from Saccharomycopsis lipolytica
}

\author{
Komakazu Gomi, ${ }^{\dagger}$ Yasuhide OTA ${ }^{*, \dagger}$ and Yasuji MinodA \\ Department of Agricultural Chemistry, Faculty of Agriculture, \\ The University of Tokyo, Bunkyo-ku, Tokyo 113, Japan \\ *Department of Applied Biochemistry, Faculty of Applied Biological Science, \\ Hiroshima University, Fukuyama, Hiroshima 720, Japan
}

Received April 11, 1986

\begin{abstract}
Three types of lipase activators $(\alpha, \beta, \gamma)$ were isolated from the culture broth of Saccharomycopsis lipolytica using high performance liquid chromatography. Activator $\gamma$ was the most active for the lipase reaction. One of them $(\beta)$ was identified with a mixture of 3,5-dihydroxy7-tetradecenoic acid and related compounds by the method of NMR and GC-MS analyses. The free carboxyl group in the compounds was essential for the activation of the lipase reaction.
\end{abstract}

In general, lipase requires an activator for triglyceride hydrolysis such as bile salts and colipase for pancreatic lipase, ${ }^{1)}$ adrenalin for the hormone-sensitive lipase from adipose tissue, ${ }^{2)}$ Apo-CII and glycerophospholipid for the lipoprotein lipase from blood, ${ }^{3)}$ and protein activator and ricinoleate tetramer for the acid lipase from castor beans. ${ }^{4)}$ Among microbial lipases, there are few enzymes that are highly regulated in the reaction of hydrolysis. The lipase from Saccharomycopsis lipolytica requires an activator for the hydrolysis of triglycerides such as liquid long-chain fatty acids (e.g. oleic acid $)^{5}$; there is an activator which is produced in the culture broth by the yeast itself. ${ }^{6)}$ This activator was partially purified and characterized by Ota $e t$ al. ${ }^{7)}$ as an acidic and oily substance at normal temperatures. To study the 'regulation of the microbial lipase, we attempted to establish the structure of the activators produced by the yeast.

In this paper we describe the isolation procedure, structure, and some characteristics of the lipase activator.

\section{MATERIALS AND METHODS}

Chemicals and preparations. The lipase used in this study was Lipase I purified from the cells of S. lipolytica by the methods, including CM-Sepharose 6B column chromatography and gel filtration, described previously. ${ }^{8)}$ Sephadex LH-20 was purchased from Pharmacia Fine Chemicals, Uppsala, Sweden. Tributyrin, butyric acid, and other chemicals were purchased from Wako Pure Chemical Industries, Ltd.

Microorganism. Saccharomycopsis lipolytica (CBS 6303, ATCC 48436, IFO 10073) used in this study was isolated from soil in 1963. $\left.{ }^{6}\right)$ This strain was at first named Candida paralipolytica as a new species, but reclassified by Yarrow $^{9)}$ into the perfect genus Saccharomycopsis in 1972.

Cultivation of the yeast. The yeast was cultivated in a jar fermentor at $30^{\circ} \mathrm{C}$ for about $12 \mathrm{hr}$. The composition of culture medium was: $2.0 \%$ glucose, $0.2 \%$ urea, $0.6 \% \quad \mathrm{KH}_{2} \mathrm{PO}_{4}, \quad 0.2 \% \quad \mathrm{~K}_{2} \mathrm{HPO}_{4}, \quad 0.1 \% \mathrm{KCl}, \quad 0.05 \%$ $\mathrm{MgSO}_{4} \cdot 7 \mathrm{H}_{2} \mathrm{O}, 0.001 \% \mathrm{FeCl}_{3} \cdot 6 \mathrm{H}_{2} \mathrm{O}, 0.1 \%$ yeast extract, and $1.0 \%$ olive oil ( $\mathrm{pH}$ 6.2). The cultivated cells were removed by continuous centrifugation $(8,000 \times g)$ and the supernatant was stored at $5^{\circ} \mathrm{C}$.

Assay of lipase activator. The activity of the lipase activator was measured by the shake $\operatorname{method}^{7)}$ on a microscale; $2 \mu \mathrm{l}$ of tributyrin, $0.4 \mu \mathrm{l}$ of a methanol solution

${ }^{\dagger}$ Present address: Technical Research Laboratory, Asahi Chemical Industry Co., Ltd., 2-1 Samejima, Fuji, Shizuoka 416, Japan.

${ }^{\dagger \dagger}$ To whom all the correspondence should be addressed. 
of the activator to be assayed, and $320 \mu$ of the following mixture were mixed in a small glass tube $(8 \times 50 \mathrm{~mm})$. The mixture was composed of $80 \mu \mathrm{l}$ of $0.2 \mathrm{M}$ Tris acid maleate$0.2 \mathrm{M} \mathrm{NaOH}$ buffer, $40 \mu \mathrm{l}$ of $0.03 \mathrm{M} \mathrm{CaCl}_{2}$ solution, and $200 \mu \mathrm{l}$ of deionized water. After heating the reaction mixture at $37^{\circ} \mathrm{C}$ for $5 \mathrm{~min}, 40 \mu \mathrm{l}$ of Lipase I solution (10 units $/ \mathrm{ml}$ ) was added and shaken continuously for $50 \mathrm{~min}$ with 180 oscillations $/ \mathrm{min}$ at $37^{\circ} \mathrm{C}$. Then $400 \mu \mathrm{l}$ of a solvent mixture (60 parts of benzene, 40 parts of isopropyl alcohol, and 2 parts of $2.4 \mathrm{~N} \mathrm{HCl}$ ) was added to the reaction mixture and shaken well to stop the lipase reaction and to extract butyric acid as a reaction product. After centrifugation $(3,000 \times g, 5 \mathrm{~min}), 2 \mu \mathrm{l}$ of the upper phase was put into a gas chromatograph (Hitachi model 163) to assay butyric acid. The conditions for gas chromatography were: column packing $5 \%$ SP-1200 on Uniport S (Gasukuro Kogyo, Inc), column size $3 \mathrm{~mm} \times 2 \mathrm{~m}$, column temperature $70^{\circ} \mathrm{C}$, carrier gas $\mathrm{N}_{2}(100 \mathrm{ml} / \mathrm{min})$, and detector FID. One unit of lipase activator was defined as the amount of activator which could liberate $1 \mu \mathrm{mol}$ of butyric acid per min under the conditions used.

High performance liquid chromatography (HPLC). For HPLC, we used a Hitachi model 5000 with a Reodyne loop injector and a Hitachi variable-wavelength ultraviolet detector; reversed phase column, a stainless steel column $(8 \times 250 \mathrm{~mm})$ packed with Nucleosil $5 \mathrm{C}_{18}$ purchased from Macherey Nagel GMBH \& Co., KG; Hitachigel column, a stainless steel column $(4.6 \times 250$ mm) packed with Hitachigel \#3013-N purchased from Hitachi Co., Ltd.; Shodex GPC column, a stainless steel column $(8 \times 500 \mathrm{~mm})$ packed with Shodex GPC AC-801 purchased from Showa Denko Co., Ltd.; mobile phase, a gradient from a mixture (methanol: water: acetic acid = $60: 40: 0.2)$ to $100 \%$ methanol for the reversed phase column, methanol containing $0.01 \%$ acetic acid for the Hitachigel column and chloroform for the Shodex GPC column.

Qualitative analysis. Anthrone-sulfate reagent which containing $50 \mathrm{mg}$ of anthrone and $1 \mathrm{~g}$ of thiourea dissolved in $100 \mathrm{ml}$ of $66 \% \mathrm{H}_{2} \mathrm{SO}_{4}$ was used for detecting sugar. Bromphenol blue reagnet $(0.04 \%)$ was used for detecting acid. Amino groups were detected with ninhydrin reagent. Phosphorous was measured by Lowry's method. ${ }^{10}$

\footnotetext{
${ }^{1} \mathrm{H}$-NMR analysis. ${ }^{1} \mathrm{H}-\mathrm{NMR}$ spectra were obtained in $\mathrm{CD}_{3} \mathrm{OD}$ on a JEOR FX-400 spectrometer at $400 \mathrm{MHz}$.
}

$G C-M S$ analysis. GC-MS spectra were taken by an LKB-9000 mass spectrometer fixed with a Hitachi gas chromatograph. Gas chromatographic conditions; $2 \%$ Silicone OV-1 on Chromosorb W AW-DMCS $(3 \mathrm{~mm} \times$ $2 \mathrm{~m}$ ), He flow rate $30 \mathrm{ml} / \mathrm{min}$, column temperature $230^{\circ} \mathrm{C}$.

Derivatization. Samples to be put on the Shodex GPC column were derivatized to methyl esters and samples to be used in GC-MS analysis were derivatized to trimethylsilyl ethers after methylation.

Methylation was done by adding etherial diazomethane to a methanolic solution of samples. Trimethylsilylation was done by adding a mixture of pyridine-bistrimethylsilyl acetamide-trimethyl chlorosilane $(5: 2: 1, \mathrm{v} / \mathrm{v})$ into the methylated samples.

Reduction was done by bubbling hydrogen gas into a methanolic solution of samples in the presence of platinum black as catalyst.

\section{RESULTS AND DISCUSSION}

\section{Isolation of lipase activators}

Three kinds of lipase activators were isolated from the culture broth of S. lipolytica and their isolation procedure was as follows.

Two hundred liters of the culture broth were concentrated to 20 liters by a vacuum evaporator and its $\mathrm{pH}$ was adjusted to 2 with hydrochloric acid. A strong acid fraction and a weak acid fraction were obtained by ether extraction; the former was named Activator $\alpha$ and the latter was separated into

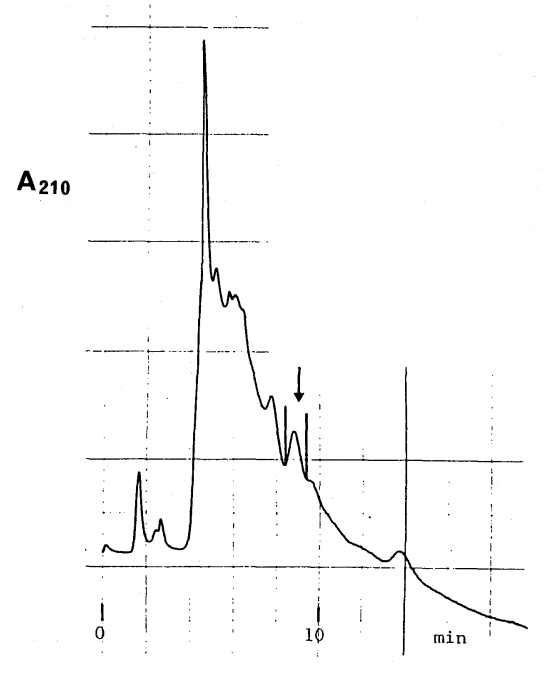

Retention time

FIG. 1. Elution Profile of Activator $\beta$ by HPLC.

Fifty microliters of methanol solution of Activator $\beta$ was injected into a Hitachigel \#3013-N column $(4.6 \times 250 \mathrm{~mm})$ and eluted with methanol containing $0.01 \%$ acetic acid at a flow rate of $1.0 \mathrm{ml} / \mathrm{min}$. The effluent was monitored by absorbance at $210 \mathrm{~nm}$ by a Hitachi UV detector. The activator activity was recognized on the peak indicated by an arrow in the figure. 
two fractions by silica gel column chromatography, which were named Activator $\beta$ and Activator $\gamma$. Activator $\beta$ was first eluted from the silica gel column $(3.8 \times 20 \mathrm{~cm})$ by a mixture of chloroform and acetone $(4: 6$ by volume) and Activator $\gamma$ by a mixture of acetone and methanol ( $7: 3$ by volume). The yields of crude Activators $\alpha, \beta$, and $\gamma$ were 910 ,
3,100 , and $870 \mathrm{mg}$, respectively. Activator $\beta$ was further purified by high performance liquid chromatography (HPLC) based on reversed phase partition or gel permeation. The chromatogram of Activator $\beta$ on a HPLC column (Hitachigel \#3013-N) is shown in Fig. 1 and the active peak assigned by an arrow was collected for the next step.

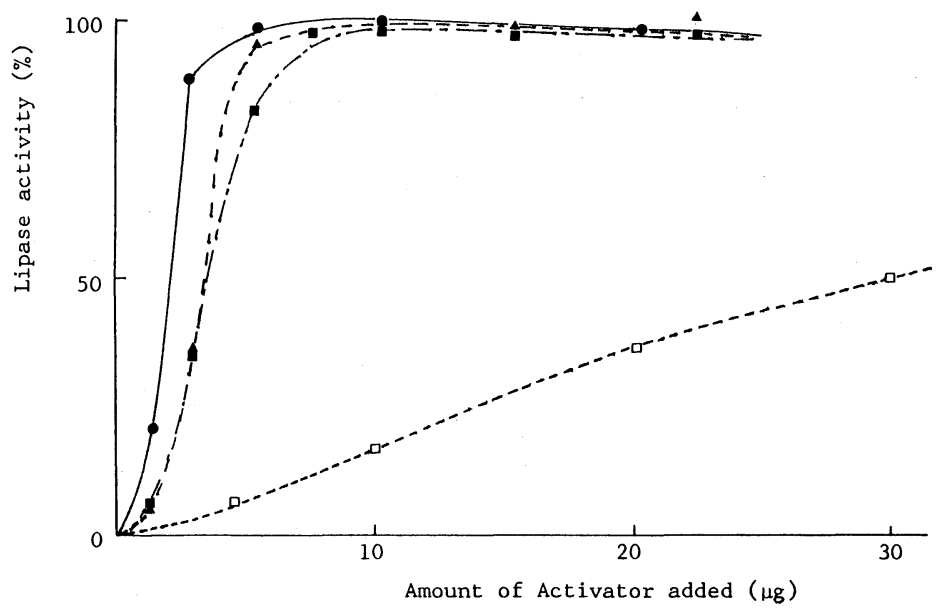

FIG. 2. Activation of Lipase Reaction by Activators $\alpha, \beta$ and $\gamma$ and Oleic Acid.

Indicated amounts of Activator were added in the reaction mixture and lipase activity was measured under the conditions described in the text.

$\boldsymbol{-} \cdot-\mathbf{\square}$, Activator $\alpha ; \boldsymbol{\Delta}--\mathbf{\Delta}$, Activator $\beta$;

Activator $\gamma ; \square--\square$, oleic acid.
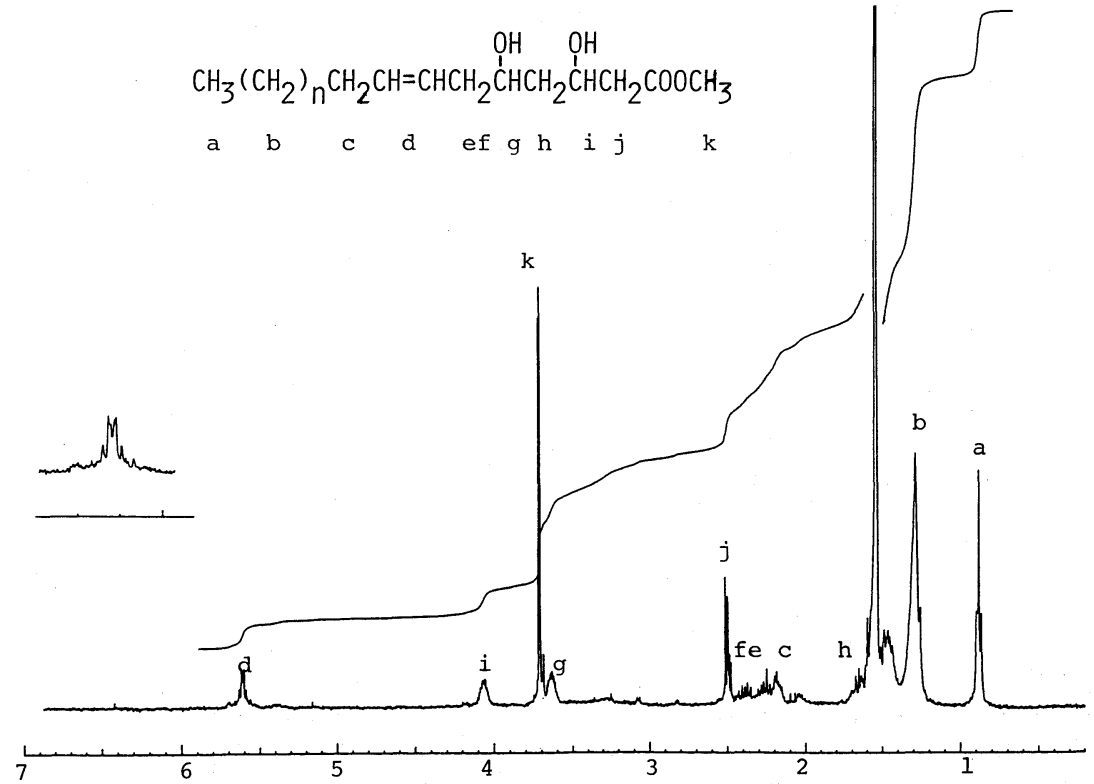

Fig. 3. ${ }^{1} \mathrm{H}-\mathrm{NMR}$ Spectrum of Activator $\beta$ (Peak 2) at $400 \mathrm{MHz}$ in $\mathrm{CD}_{3} \mathrm{OD}$. 


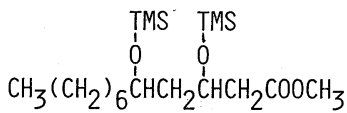

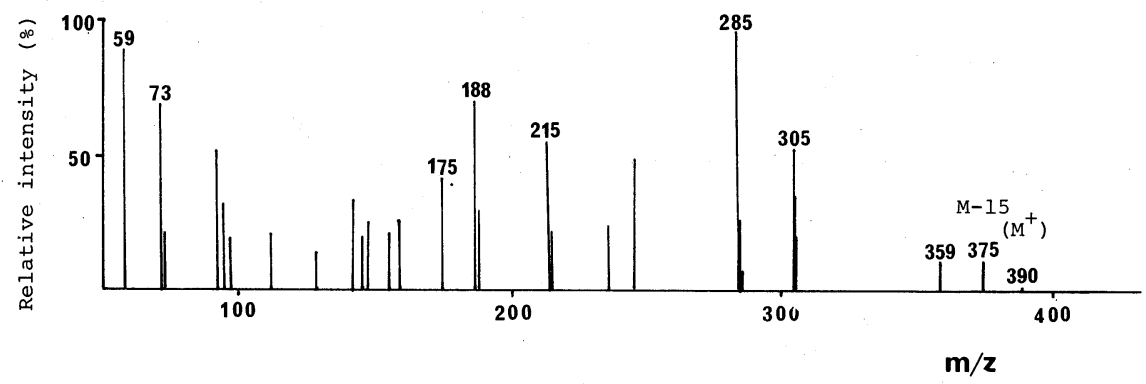

(a)

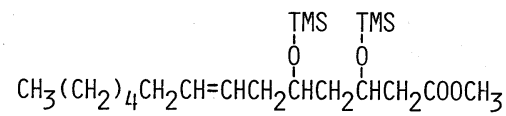

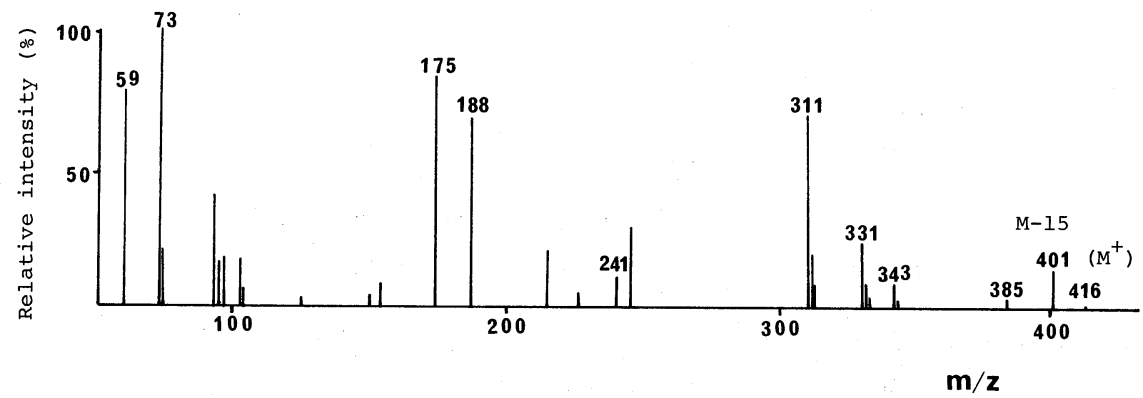

(b)<smiles>CCCCCCC(C)CC(C)=O</smiles>

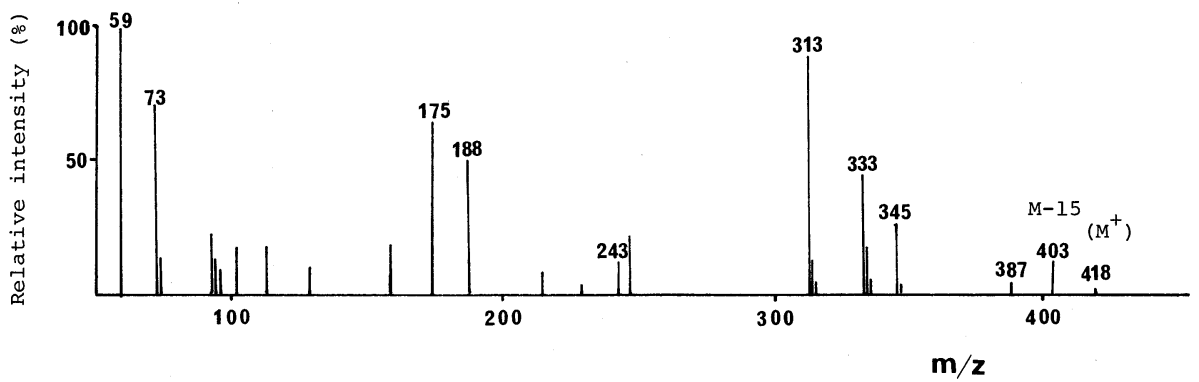

(c)

FIG. 4. Mass Spectrum of Activator $\beta$ (Peak 2).

The gas chromatogram of the trimethylsilyl derivative of peak 2 (one of the two peaks obtained by Shodex GPC AC-801 HPLC of methylated Activator $\beta$ ) gave two peaks (peaks A and B). The trimethylsilyl derivative of reduced peak 2 also gave two peaks (peaks A and $B^{\prime}$ ) by gas chromatography. (a), (b) and (c) are mass spectra of peaks A, B, and $\mathrm{B}^{\prime}$ respectively. 
The activation çurve by three kinds of activators for the hydrolysis of tributyrin is shown in Fig. 2. The lipase reaction was more strongly activated by these activators produced by the yeast than by other synthetic activators such as oleic acid.

\section{Identification of Activator $\beta$}

1) Qualitative analysis. The fraction of Activator $\beta$ eluted from the Hitachigel column was used in the following reactions. Ninhydrin, bromphenol blue (BPB), or anthronesulfuric acid reagent was sprayed on spots on pre-coated silica gel plates. Only the BPB test gave a postive result. No phosphorus was detected by Lowry's method. ${ }^{10)}$ From these findings it is suggested that Activator $\beta$ is neither phospholipid nor glycolipid but a simple lipid which may have a carboxyl group.

2) ${ }^{1} H-N M R$ analysis of Activator $\beta$. The same fraction described above was dissolved in methanol and methylated with diazomethane dissolved in ether. The methylated derivative was dissolved in chloroform and put on a HPLC column (Shodex GPC AC-801). Its elution profile gave two peaks (peaks 1 and 2 ), the molecular weights of which were about 350 and 250 , respectively.

The $400 \mathrm{MHz}{ }^{1} \mathrm{H}-\mathrm{NMR}$ spectrum of peak 2 taken in $\mathrm{CD}_{3} \mathrm{OD}$ (Fig. 3) suggests that it has carboxyl and hydroxyl groups, and double bonds. Spin decoupling experiments suggest the structure of Activator $\beta$ as shown in Fig. 3.

3) GC-MS analysis of Activator $\beta$. The gas chromatogram of the trimethylsilyl derivative of peak 2 gave two peaks of which the retention times were 5.2 and $11.0 \mathrm{~min}$ respectively under the conditions used. The mass spectra of the former (peak A) and the latter (peak B) gave the molecular ion at 390 and $416 \mathrm{~m} / \mathrm{z}$ respectively, as shown in Figs. 4(a) and (b). The peak 2 fraction was reduced by bubbling hydrogen using platinum black as catalyst and the trimethylsilyl derivative of reduced peak 2 fraction was used in GC-MS analysis. The gas chromatogram of this derivative also gave two peaks and their mass spectra gave the molec-
Table I. EfFects of Chemical Modification of Activators ON Lipase ReAction

To the reaction mixture were added $7.5 \mu \mathrm{g}$ of Activator $\alpha, 5 \mu \mathrm{g}$ of Activator $\beta$, or $2.5 \mu \mathrm{g}$ of Activator $\gamma$, as described in the text. Lipase activity is expressed as the percentage of those with non-modified Activators.

\begin{tabular}{lccc}
\hline \multicolumn{1}{c}{ Activator } & $\alpha$ & $\beta$ & $\gamma$ \\
\hline Non-modified & $100 \%$ & $100 \%$ & $100 \%$ \\
Methylated & 29 & 7 & 10 \\
Reduced & 58 & 42 & 18 \\
\hline
\end{tabular}

ular ion at 390 and $418 \mathrm{~m} / z$ (Fig. 4(c)) respectively. From the results of ${ }^{1} \mathrm{H}-\mathrm{NMR}$ and GCMS analyses it is considered that the peak 2 fraction contained two organic acids, 3,5dihydroxy-7-tetradecenoic acid and 3,5-dihydroxydodecanoic acid.

The GC-MS analysis of peak 1 eluted from the Shodex GPC column suggests that it contained 3,5-dihydroxyhexadecenoic acid and 3,5-dihydroxyoctadecenoic acid. Therefore we conclude that Activator $\beta$ was unsaturated and saturated 3,5-dihydroxy acids of which the carbon number ranged from 12 to 18 .

The structure of Activator $\gamma$ is much more interesting because its activity was the strongest among the activators; however, our attempts to isolate it have not been successful. The molecular weight of Activator $\gamma$ was larger than Activator $\beta$, probably more than 500, judging from the elution profile with the Shodex GPC column. Activator $\gamma$ may also have carboxyl and hydroxyl groups, and double bonds, as shown by qualitative analysis and ${ }^{1} \mathrm{H}-\mathrm{NMR}$ spectrum.

\section{Chemical modification of Activators}

Crude Activators $\alpha, \beta$ and $\gamma$ were methylated by diazomethane or reduced by hydrogen gas. Then the activity of each modified Activator for the hydrolysis of tributyrin was measured by adding a minimum dose of it to the reaction mixture (Table I). From this result it is suggested that the carboxyl group is important in the activation of lipase.

Acknowledgments. We wish to express our sincere 
thanks to Dr. A. Isogai and Dr. I. Yamaguchi (Department of Agricultural Chemistry, Faculty of Agriculture, the University of Tokyo) for their useful advice and discussion throughout this work.

\section{REFERENCES}

1) M. Sémériva and P. Desnuelle, Adv. Enzymol., 48, 319 (1979).

2) H. Okuda, T. Tsujita, M. Sumida, Y. Takahashi, D. Shimizu and S. Fujii, J. Biochem. (Tokyo), 93, 575 (1983).

3) L. C. Smith and A. M. Gotto, Jr., Ann. Rev. Biochem., 47, 751 (1974).
4) R. L. Ory and B. Borgström, Biochim. Biophys. Acta, 212, 521 (1970).

5) Y. Ota, T. Nakamiya and K. Yamada, Agric. Biol. Chem., 36, 1895 (1972).

6) K. Yamada and Y. Ota, Nippon Nôgeikagaku Kaishi, 37, 649 (1963).

7) Y. Ota, K. Yoshioka, Y. Minoda and K. Yamada, Agric. Biol. Chem., 37, 2879 (1973).

8) Y. Ota, K. Gomi, S. Kato, T. Sugiura and Y. Minoda, Agric. Biol. Chem., 46, 2885 (1982).

9) D. Yarrow, Antonie van Leeuwenhock, 38, 357 (1972).

10) R. R. Lowry and I. J. Tinsley, Lipids, 9, 491 (1974). 